\title{
Metabolite Profiling Reveals Predictive Biomarkers and the Absence of $\beta$-Methyl Amino-L-Alanine in Plasma from Individuals Diagnosed with Amyotrophic Lateral Sclerosis
}

\author{
*Michael S. Bereman ${ }^{1,2,3}$, Kaylie I. Kirkwood ${ }^{2}$, Tharani Sabaretnam ${ }^{4}$, Sarah \\ Furlong ${ }^{4}$, Dominic B. Rowe ${ }^{4}$, Gilles J. Guillemin ${ }^{4}$, Allyson L. Mellinger ${ }^{2}$, David \\ C. Muddiman ${ }^{2,3,5}$ \\ ${ }^{1}$ Department of Biological Sciences, ${ }^{2}$ Department of Chemistry, ${ }^{3}$ Center for \\ Human Health and the Environment, 5 Molecular Education, Technology and \\ Research Innovation Center, North Carolina State University Raleigh NC 27695 \\ ${ }^{4}$ Medicine and Health Sciences, Macquarie University, NSW, 2109 Australia
}

\author{
*Author for Correspondence \\ Michael S. Bereman, Ph.D. \\ Department of Biological Sciences \\ Center for Human Health and the Environment \\ North Carolina State University \\ Raleigh, NC 27606 \\ Phone: 919.515.8520 \\ Email: msberema@ncsu.edu
}




\section{TABLE OF CONTENTS}

Figure S1. BMAA calibration curves

Figure S2. Quality control boxplots

Figure S3. Comparison of the distribution of metabolite abundances and PCA analysis

Figure S4. Comparison of model performance in separating disease and control sets

Figure S5. Statistical analysis of model performance

Figure S6. Variable importance for rate of disease progression

Figure S7. Electropherogram of solvent extracted metabolites (BMAA absent)

Figure S8. Electropherogram from protein hydrolysis (BMAA absent)

Figure S9. Detection of BMAA from positive control

Supplemental File 1. Metabolite and QC data (separate file)

Supplemental File 2. Abundance distributions of metabolites (separate file)

Supplemental File 3. Correlation between metabolites ALS progression (separate file)

Supplemental File 4.. Abundance distributions by disease progression (separate file) 
Figure S1. Calibration curves of peak area ratio of BMAA/SIL-BMAA vs. concentration of BMAA ( $\mathrm{ng} / \mathrm{mL}$ ) injected for analysis, using A) hydrolyzed human plasma protein as complex background and B) extracted human plasma metabolites as complex background with SIL-BMAA spiked in a constant concentration for quantitation of BMAA.

a

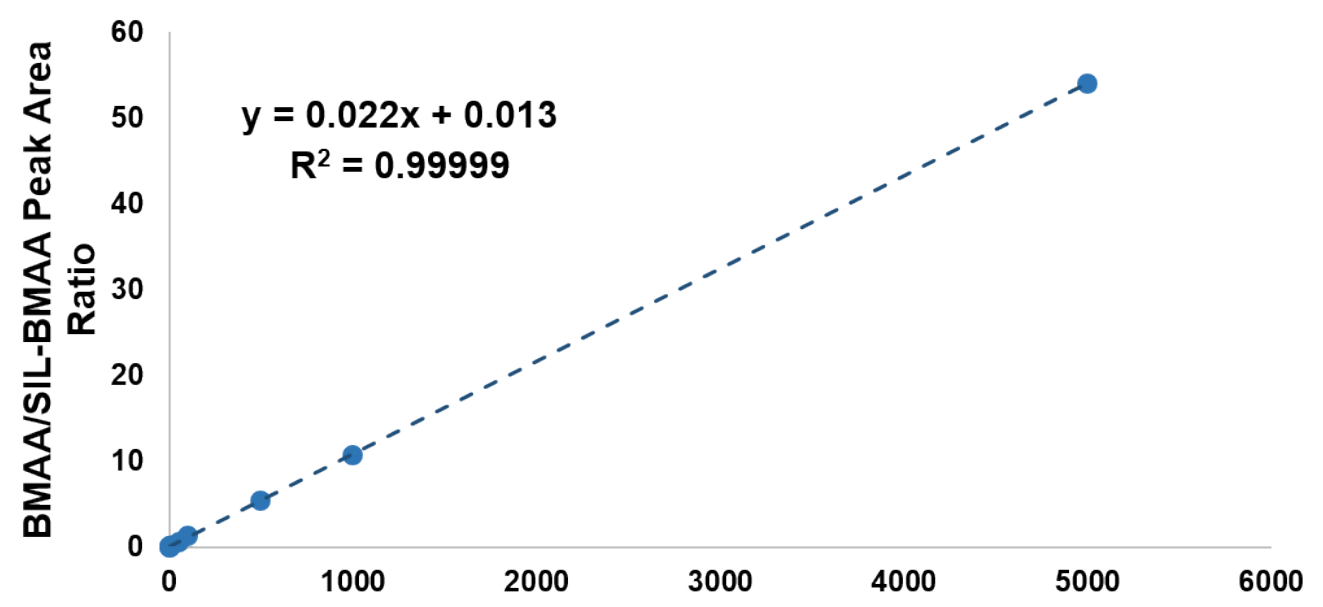

b

[BMAA] Injected on Chip $(\mathrm{ng} / \mathrm{mL})$

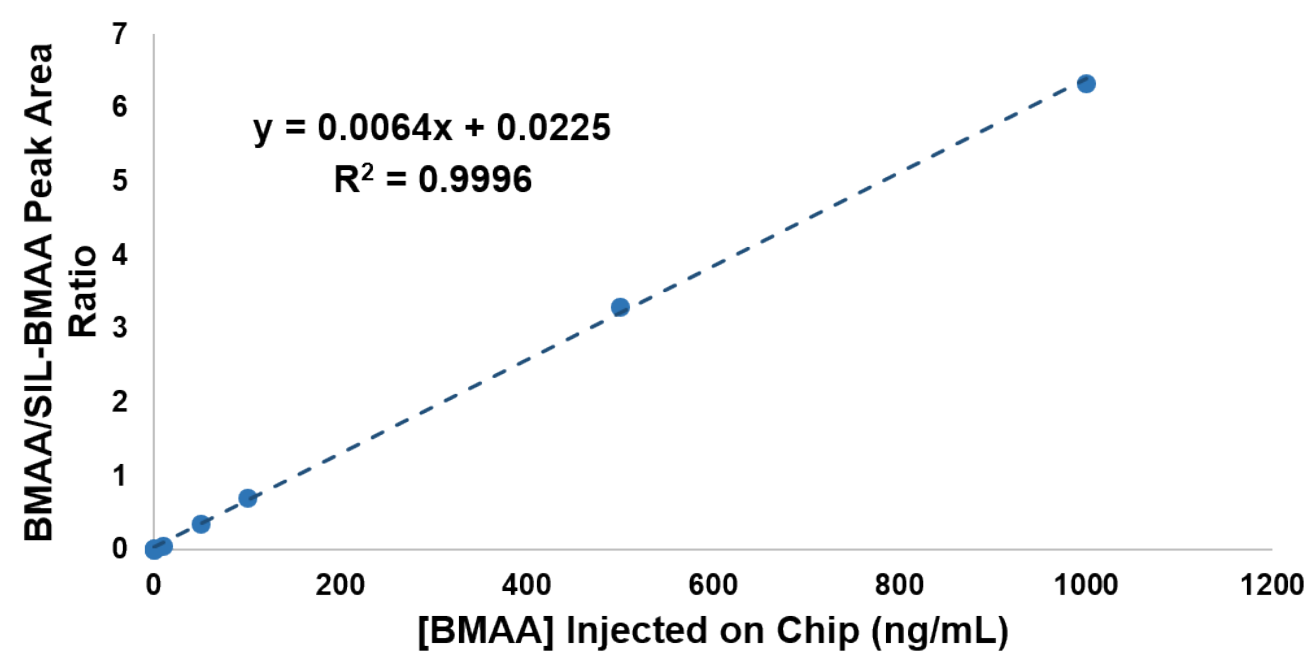


Figure S2. Boxplots of the precision of quantitation within each chip and the technical variance (TV) in sample preparation for each batch. Overall experimental reproducibility was calculated from the pooled QC that was prepared once each batch.

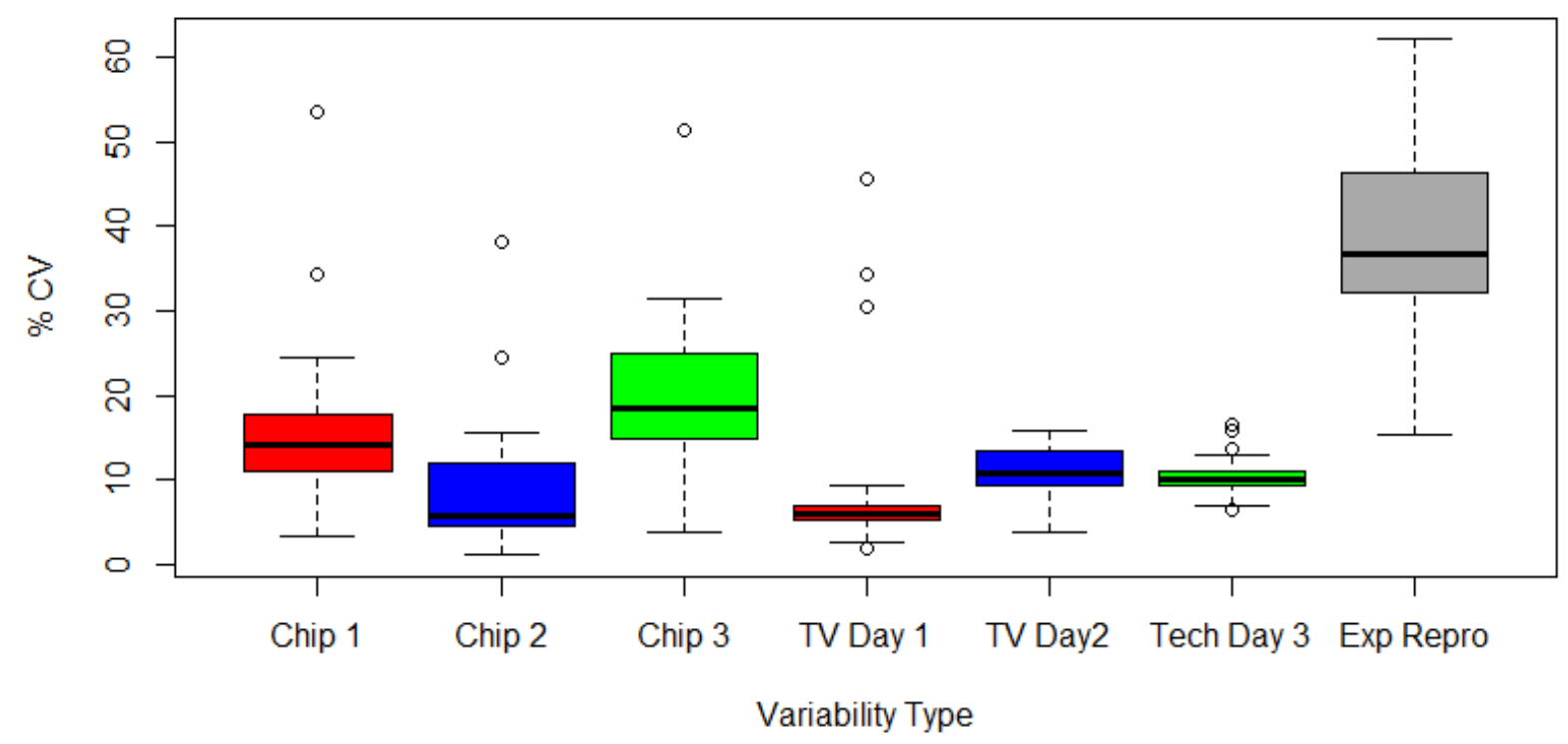


Figure S3. Comparison of overall metabolite abundance between A) Control vs. ALS and B) males vs females. K-means clustering in principal component space reveals no discernible groupings between C) Controls and ALS D) males vs. females.
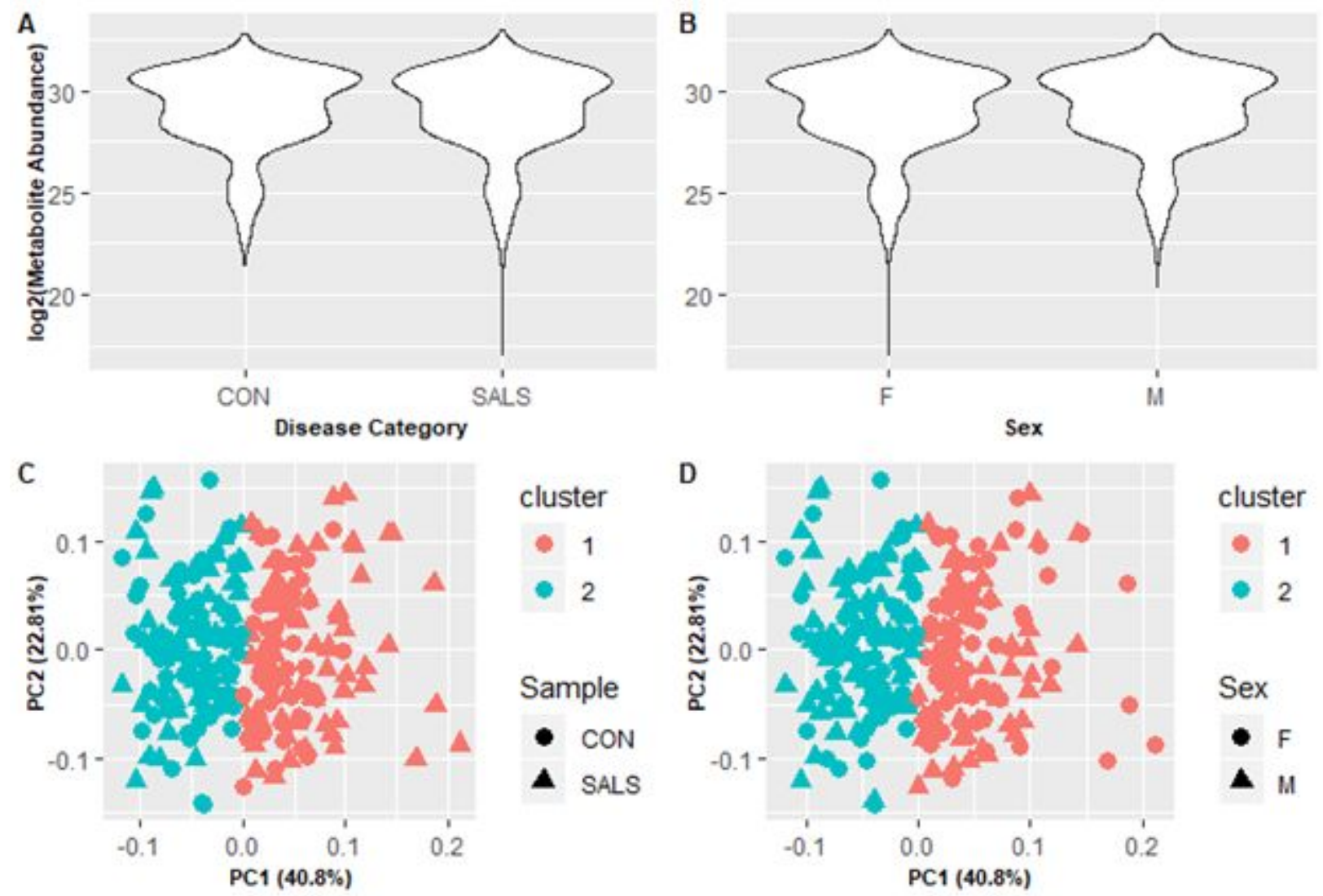
Figure S4. Comparison of classifier performance in designation of disease status. The linear model (LDA) statistically outperformed the other two nonlinear models as summarized below. The reduced feature LDA model had identical performance as the larger feature model based on AUC.

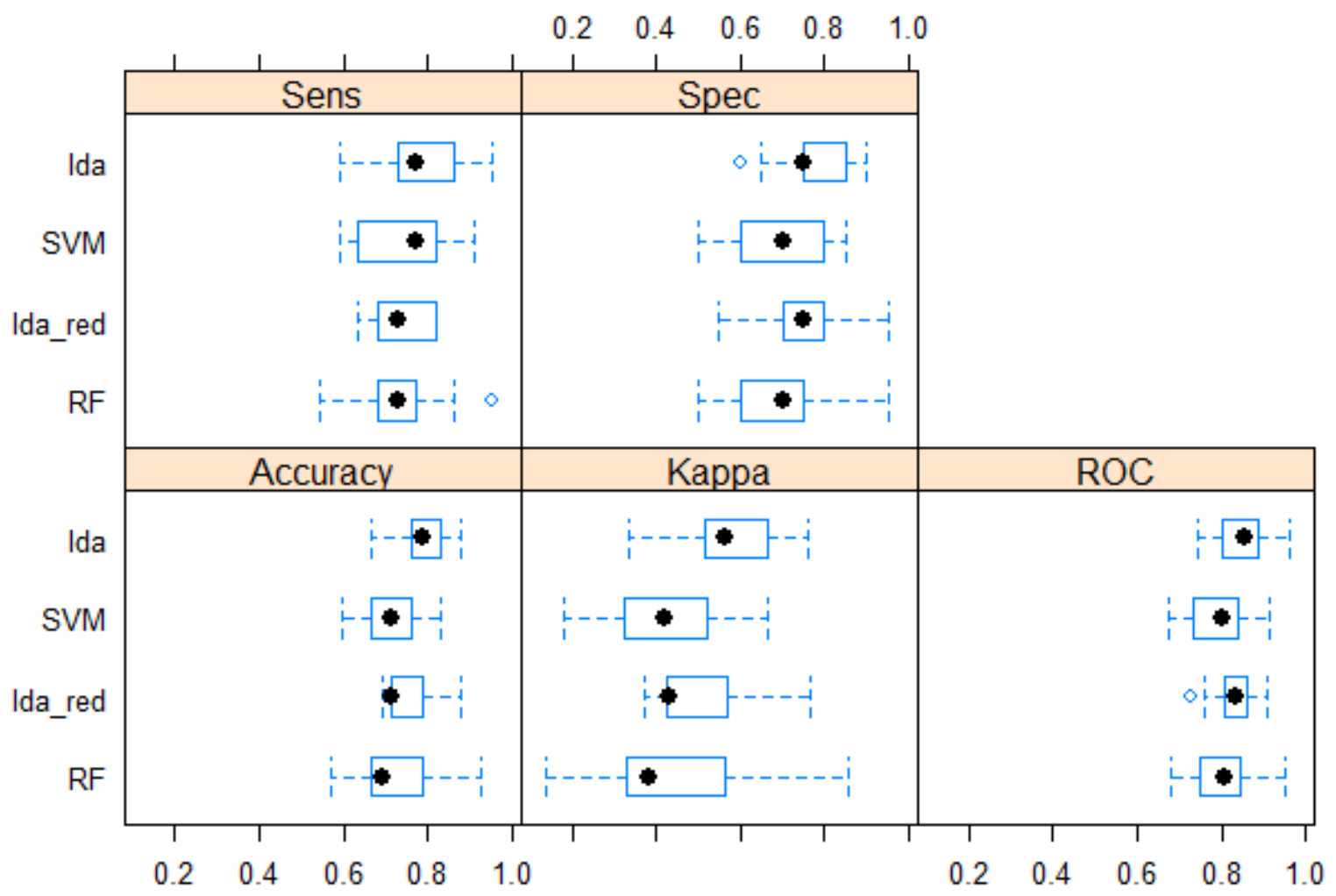


Figure S5. Results from the statistical analysis amongst each model's performance on the resampled data sets.

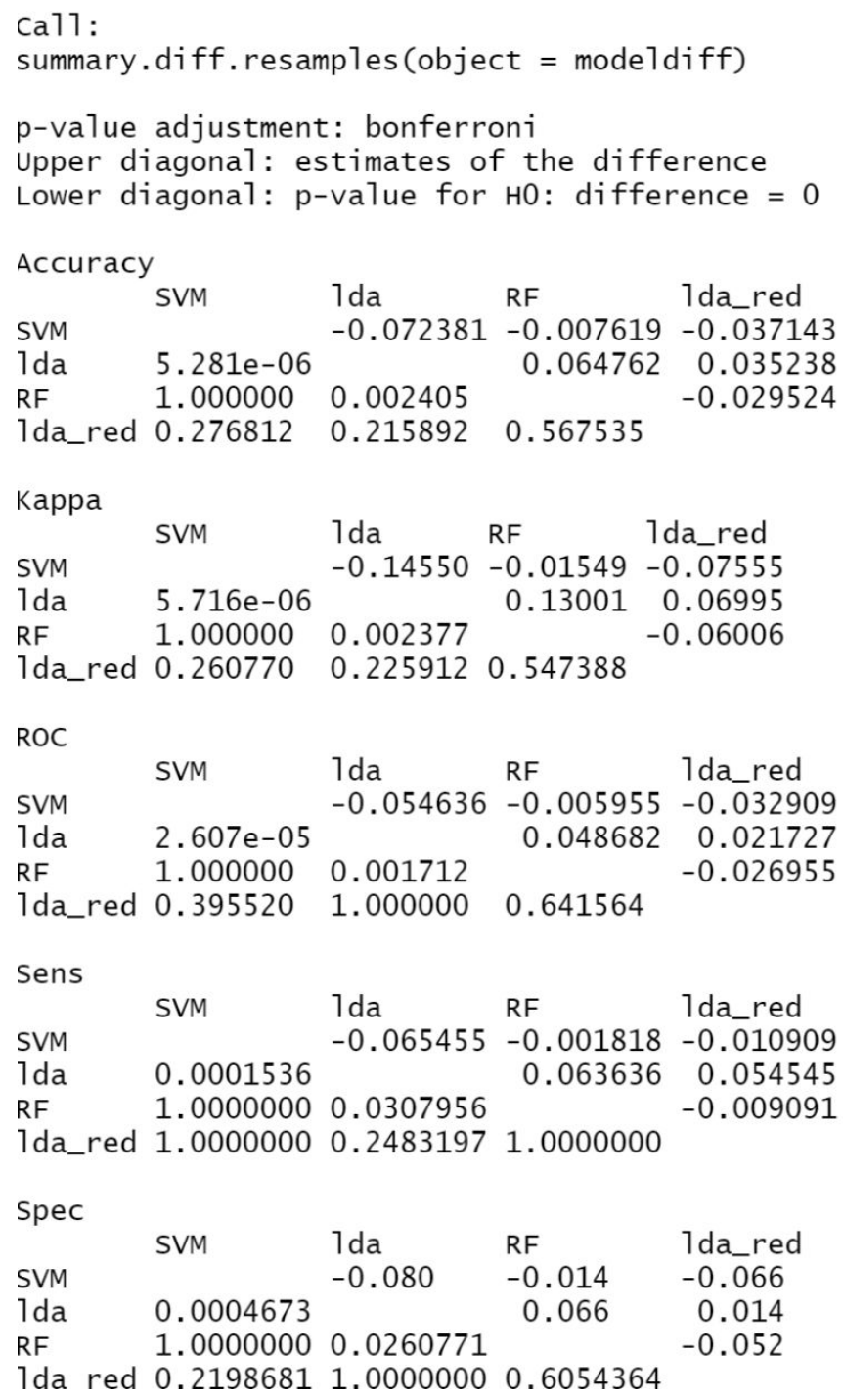


Figure S6. The average performance, based on the area under the curve, for each metabolite in determination of categories of disease progression (Blue). For comparison, the variable importance is shown for the randomized data set (Red).

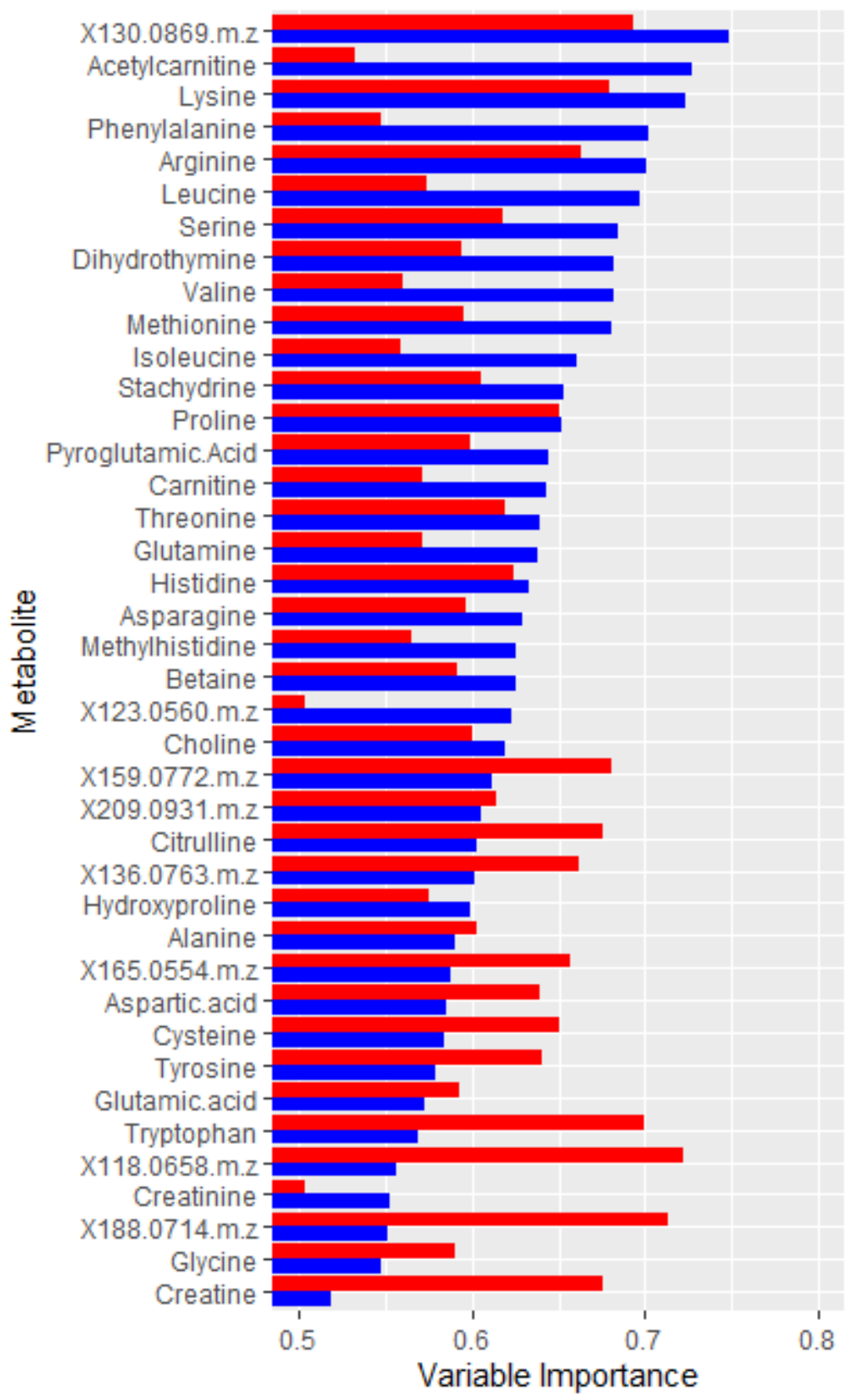


Figure S7. A representative A) Base peak electropherogram for human plasma following solvent extraction and B) Base peak electropherogram zoomed into the region of interest for BMAA detection. Representative extracted ion electropherograms for C) BMAA $(119.0815 \mathrm{~m} / \mathrm{z})$ and D) SIL-BMAA $(124.0856 \mathrm{~m} / \mathrm{z})$ showing the absence of free BMAA from extracted human plasma.
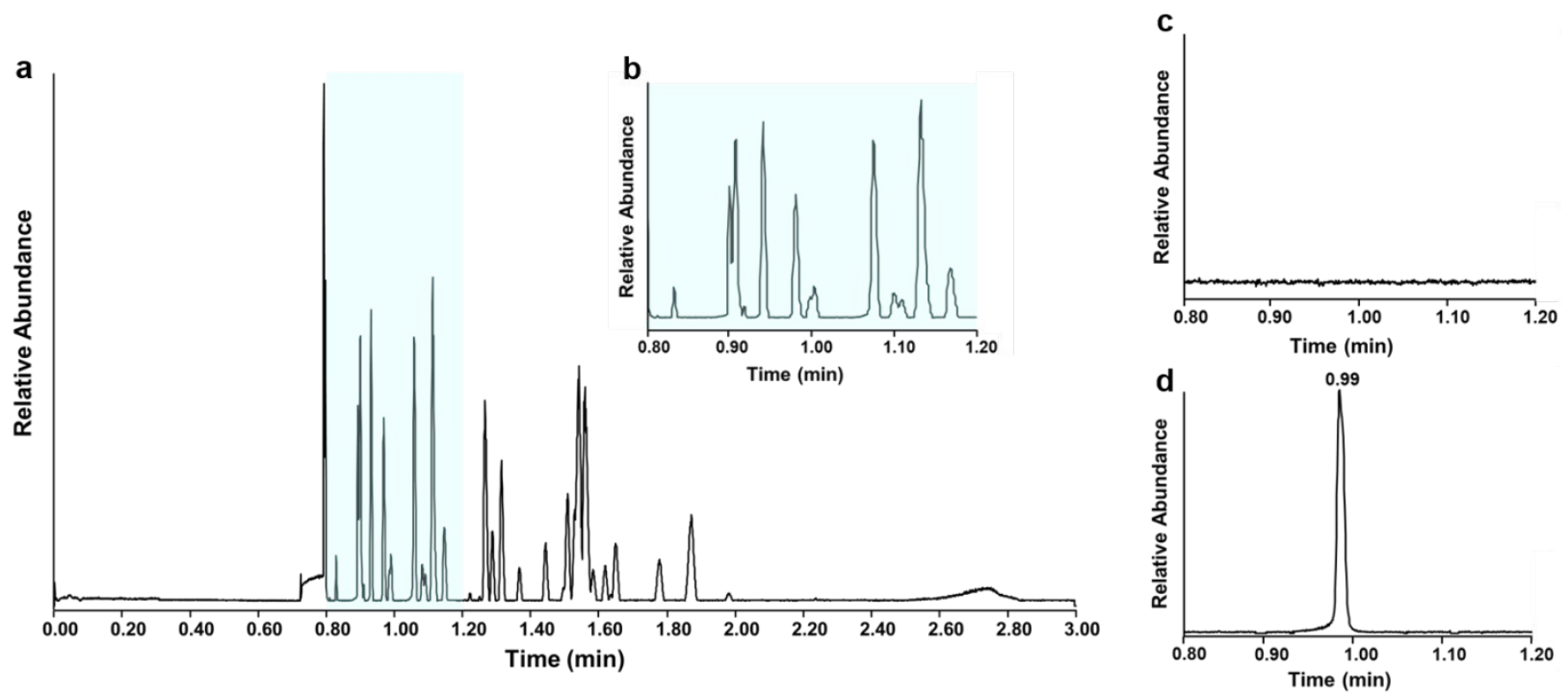
Figure S8. Representative A) Base peak electropherogram for human plasma following hydrolysis and B) Base peak electropherogram zoomed into the region of interest for BMAA detection. Representative extracted ion electropherograms for C) BMAA $(119.0815 \mathrm{~m} / \mathrm{z})$ and D) SIL-BMAA $(124.0856 \mathrm{~m} / \mathrm{z})$ showing the absence of protein bound BMAA from hydrolyzed human plasma.
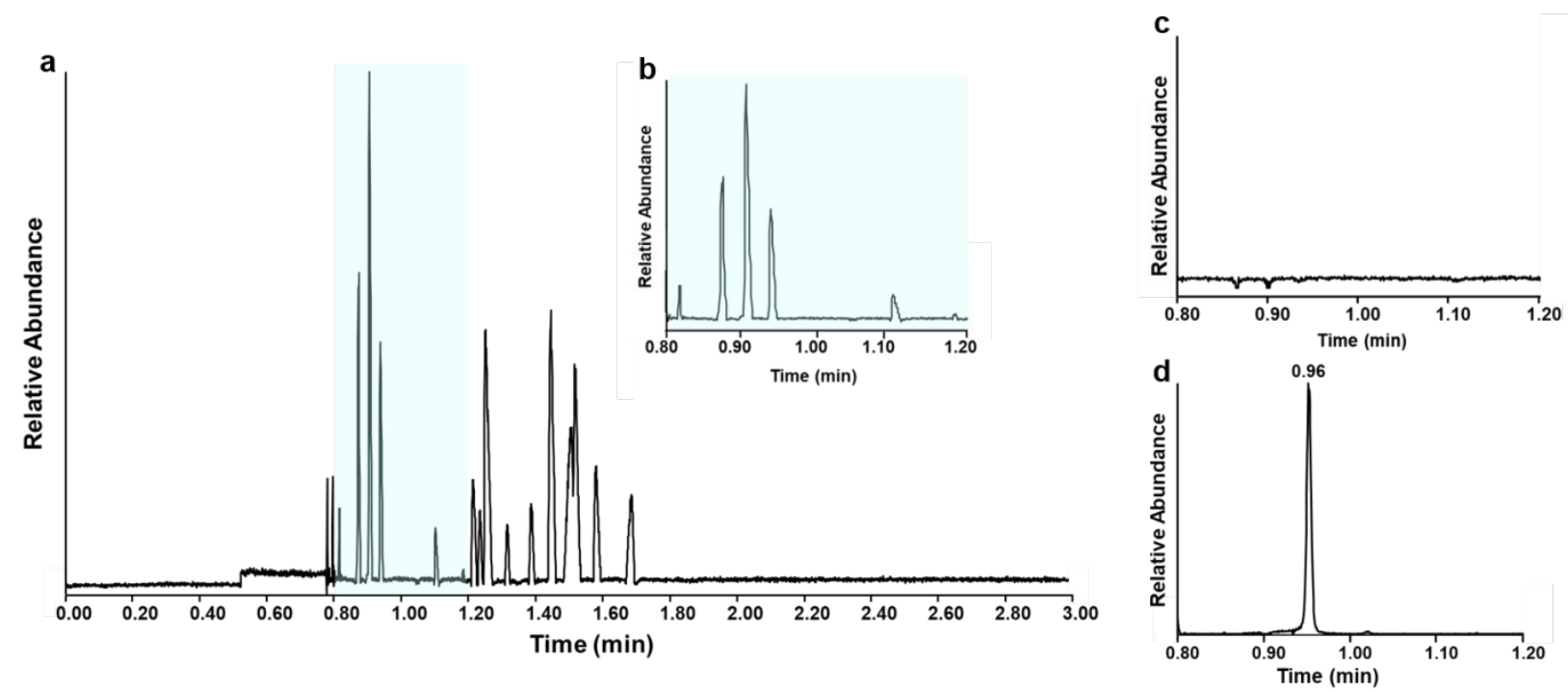
Figure s9. Detection of BMAA in a representative positive control sample comprised of pooled plasma with a synthetic BMAA-incorporated protein following acid hydrolysis. Fragment ion electropherograms extracted from targeted MS/MS scans in Skyline for A) BMAA and B) SIL-BMAA showing co-migration and detection of all five qualifying product ions.
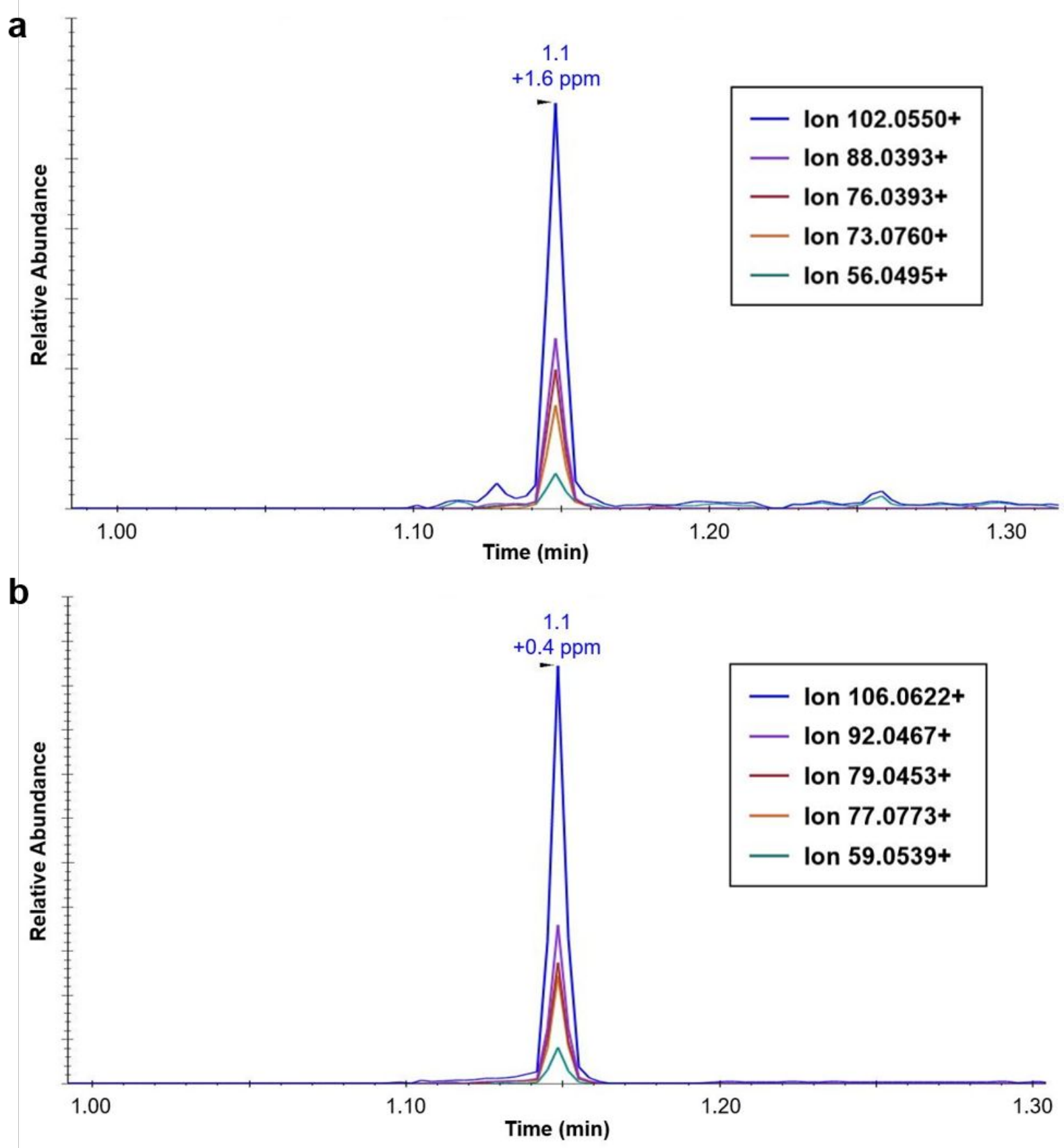